\title{
Vibration Measurement and Suppression for Laser Galvanometers Using a Micro-electromechanical System-based Accelerometer
}

\author{
Po-Huan Chou, ${ }^{1}$ Yu-Liang Hsu, ${ }^{2 *}$ Shih-Chin Yang, ${ }^{3}$ Hsing-Cheng Chang, ${ }^{2}$ \\ Yu-Chen Kuo, ${ }^{2}$ Li-Feng Chiu, ${ }^{2}$ and Yu-Tai Chen ${ }^{2}$

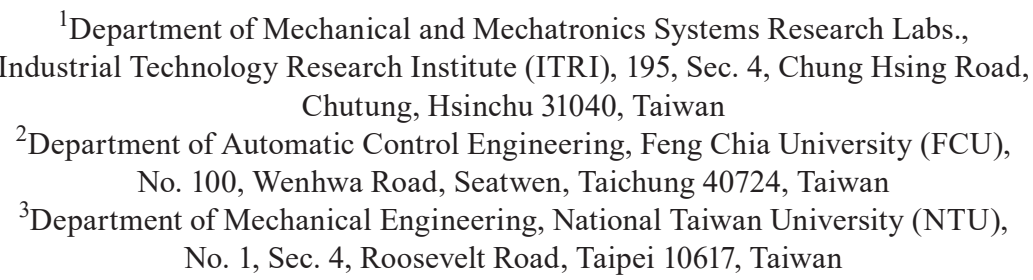 \\ (Received January 18, 2018; accepted July 3, 2018)
}

Keywords: galvanometer, MEMS-based accelerometer, vibration analysis, analog comb filter, vibration suppression

In this study, we develop a vibration measurement, analysis, and suppression system for laser galvanometers. A galvanometer control system integrates a notebook computer, an intelligent motion control platform (IMP-2), a servo driver circuit, and a galvanometer driver module for driving laser galvanometers. The proposed vibration measurement, analysis, and suppression system composed of a micro-electromechanical system (MEMS) piezoelectric accelerometer, the fast Fourier transformation (FFT) algorithm operated on a notebook computer, and an analog comb filter circuit is utilized to measure, analyze, and suppress vibrations generated from the mirror-installed galvanometer motors during rotation motions, respectively. First, we use the MEMS-based accelerometer to measure and record the vibration signals from the mirror-installed galvanometer motors during rotation motions. Second, we carry out the frequency spectrum analysis of vibration acceleration signals using the FFT algorithm. Subsequently, we design an efficient analog comb filter on the basis of the observed harmonic or resonance frequencies for suppressing the galvanometer vibrations. Finally, on the basis of the experimental results, we successfully validate that the proposed inertial-sensing-based vibration measurement, analysis, and suppression system can measure and analyze the galvanometer vibrations, and suppress them effectively to improve the accuracy and stability of the laser galvanometer scanning systems.

\section{Introduction}

With the rapid development of laser machining technology, laser machining systems with galvano scanners have become indispensable in laser processing industrial techniques such *Corresponding author: e-mail: hsuyl@fcu.edu.tw https://doi.org/10.18494/SAM.2018.1976 
as welding, cutting, sculpting, drilling, marking, mode filling, and surface treatment. ${ }^{(1-6)}$ The laser machining systems with galvano scanners can be used to effectively replace the traditional machinery machining approach according to the following advantages: (1) noncontact treatment, (2) absence of pollution, (3) high movement speed, (4) prevention of tool wear, (5) high precision, and (6) high efficiency. In addition, the laser galvanometer scanning systems are widely utilized in material processing because they can control the laser spot more accurately and rapidly than other scanning systems for producing a small and uniform laser spot size in an objective flat field. The control architecture of a laser galvanometer scanning system is composed of a laser light source, $\mathrm{X}$ and $\mathrm{Y}$ scanning mirrors, galvanometer motors, and driver controllers. Galvanometer motors are a type of voice coil motor, which is a sensitive electromechanical actuator. They work on the principle of the interaction effects of the stator magnetic field and the magnetic field generated from the current passing through the permanent magnet, which can provide sufficient torque for the rotation of the rotor and further control of the rotation angles of the $\mathrm{X}$ and $\mathrm{Y}$ scanning mirrors. The advantages of galvanometer motors are high rotational inertia ratio, high position accuracy, high stability, low time constant, fast response, and high position repeatability. However, galvanometer motors suffer from limited rotation angles and poor heat dissipation. In addition, the harmonic or resonance frequency, increased temperature, and high-frequency noise decrease the precision and stability of laser galvanometer scanning systems, which are induced by the imbalanced rotations generated from the high-speed swing motions of galvanometer mirrors. Among the above-mentioned deterioration factors, the harmonic or resonance frequency is a very important factor for galvanometer motors. If the harmonic or resonance frequency is not suppressed by a proper vibration suppression strategy, the coil of galvanometer motors might be burned out. Hence, the precision and stability of laser galvanometer scanning systems can be effectively enhanced by accurately detecting and analyzing the harmonic or resonance frequencies induced by the imbalanced rotations of galvanometer mirrors, and designing a suitable vibration suppression strategy for suppressing the harmonic or resonance frequencies.

In recent years, micro-electromechanical system (MEMS)-based accelerometers have become a popular solution for monitoring vibrations in industry applications, since they are inexpensive, small, lightweight, and have low power consumption. ${ }^{(7-17)}$ To name a few, Maruthi and Hegde used a MEMS accelerometer to detect and analyze vibrations generated from multiple faults in roller element bearings of a three-phase induction motor under no load, single phase, and unbalanced voltage conditions. The experimental results demonstrate that the fault frequencies in the motor vibration spectrum can be determined by fast Fourier transform (FFT) and used to diagnose multiple faults in the bearings. ${ }^{(12)}$ Huang and Chen developed a dual-axis MEMS accelerometer with a full scale range of $\pm 50 \mathrm{~g}$ and a bandwidth of $5 \mathrm{kHz}$ to monitor the vibration of machine tools, which integrates two MEMS accelerometer chips into a low-pass filter circuit. The experimental results validate that the dual-axis MEMS accelerometer is more suitable than the lead zirconate titanate (PZT) accelerometer for measuring the vibration of machine tools at a low cost. ${ }^{(13)}$ Linessio et al. implemented, characterized, calibrated, and tested a biaxial optical fiber accelerometer with a high signal-to-noise ratio (SNR) for monitoring vibration signals in three-phase induction motors. ${ }^{(14)}$ Son et al. utilized MEMS-based 
accelerometers and current sensors to measure vibration and current signals, and then used the kernel principal component analysis (KPCA) to obtain 25 principal components as salient fault signatures of induction motor failures from the statistical values extracted from both signals. The selected salient fault signatures can be used as inputs for one-against-all multiclass support vector machines (OAA MCSVMs), a random forest (RF), and a fuzzy k-nearest neighbor (Fk-NN) to classify various induction motor failures. The classification rate of the OAA MCSVMs was found to be higher (100\%) than those of the two classifiers ( $86 \%$ for RF and $80 \%$ for Fk-NN). ${ }^{(15)}$

In this paper, we present a vibration measurement, analysis, and suppression system for laser galvanometers during rotation motions. A galvanometer control system comprises a notebook computer, an intelligent motion control platform (IMP-2), a servo driver circuit, and a galvanometer driver module to drive laser galvanometers. The proposed vibration measurement, analysis, and suppression system is utilized to measure, analyze, and suppress the vibrations resulting from the galvanometer motors during rotation motions; such a system is composed of a MEMS-based piezoelectric accelerometer, the FFT algorithm operated on a notebook computer, and an analog comb filter circuit. First, the MEMS-based accelerometer is used to measure and record the vibration signals generated from the galvanometer motors during rotation motions. Subsequently, the FFT algorithm is utilized to determine the vibration frequencies of the galvanometer motors. Finally, according to the observed harmonic or resonance frequencies, the efficient analog comb filter circuit is designed for suppressing the galvanometer vibrations. The main contribution of this paper is the design of a proper vibration suppression strategy to effectively restrain the harmonic or resonance frequencies based on the FFT of the vibration signals measured using the MEMS-based accelerometer, which can further effectively enhance the precision and stability of the laser galvanometer scanning systems.

The rest of this paper is organized as follows: In Sect. 2, the galvanometer system architecture is described in detail. The proposed vibration measurement, analysis, and suppression system is described in Sect. 3. Section 4 presents the experimental results. Finally, the conclusions are given in Sect. 5.

\section{Galvanometer System Architecture}

The architecture of a laser galvanometer scanning system is mainly composed of a laser light source, $\mathrm{X}$ and $\mathrm{Y}$ scanning mirrors, galvanometer motors, and driver controllers. In general, the precision of laser machining will be influenced by the power and wavelength of the laser light source, the $\mathrm{X}$ and $\mathrm{Y}$ scanning mirror mechanisms, and the rotation angles controlled by the galvanometer motors. The operation processing is described as follows: (1) Users input a defined pattern into a computer and then transfer the pattern to voltage signals to drive the galvanometer motors with the $\mathrm{X}$ and $\mathrm{Y}$ scanning mirrors for generating rotation motions. (2) The laser light generated from the laser light source can be scanned by the rotation motions of the $\mathrm{X}$ and $\mathrm{Y}$ scanning mirrors, and the rotation angles of the mirror-installed galvanometer motors will be changed along with the varying driving voltage signals. (3) The laser light focuses on focal points based on the reflection rotation angles of the scanning mirrors to form the user-defined patterns in the objective flat field. The architecture of the laser galvanometer scanning system is shown in Fig. 1. 


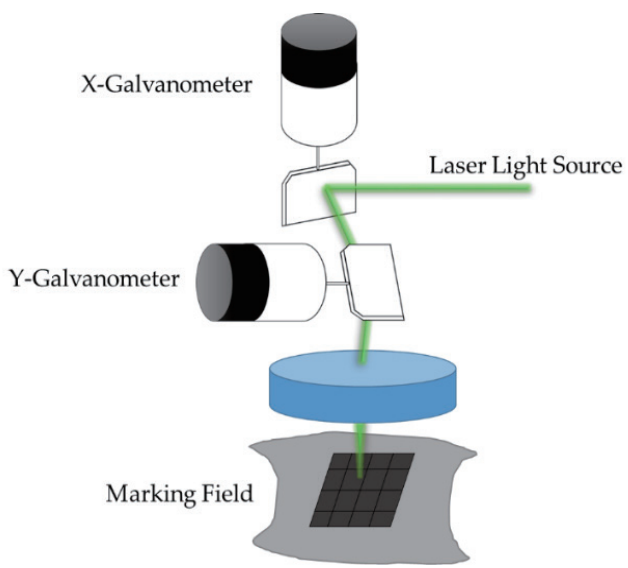

Fig. 1. (Color online) Architecture of a laser galvanometer scanning system.

The galvanometer control system presented in this paper consists of a notebook computer, an intelligent motion control platform (IMP-2), a servo driver circuit, a galvanometer driver module, and a mirror-installed galvanometer motor. An overview of the galvanometer control system for driving the mirror-installed galvanometer motor is illustrated in Fig. 2. We describe each component in detail as follows: (1) Notebook computer: The ttermpro program was run on a notebook computer running Microsoft Windows 10 operating system with a Core i5-4460 3.2 GHz CPU and $8 \mathrm{~GB}$ memory for setting the waveforms and frequencies of the command signals to the intelligent motion control platform (IMP-2). (2) Intelligent motion control platform (IMP-2): The IMP-2 produced by the Industrial Technology Research Institute (Hsinchu, Taiwan) is used to provide digital command signals for driving the mirror-installed galvanometer motor. The IMP-2 is equipped with a built-in microprocessor (PowerPC 440) and a real-time operating system (VxWorks RTOS), and integrates PCI-Bus, Ethernet (A+PC mode), and RS232 (standalone mode) interfaces. (3) Servo driver circuit: It is used to convert the digital command signals transmitted from the IMP-2 into analog command voltage signals for driving the galvanometer motor. (4) Galvanometer driver module: This module composed of a proportional-integral-derivative (PID) controller and a motor driving circuit is used to receive the analog command voltage signals generated from the servo driver circuit and send the control signals to the galvanometer motor for controlling the rotation angles of the scanning mirror. (5) Mirror-installed galvanometer motor: The galvanometer motor $(6220 \mathrm{H})$ with a $5 \mathrm{~mm}$ mirror produced by Cambridge Technology (MA, USA) can receive the control signals and generate the rotation angles of the scanning mirror to scan the laser light to form user-defined patterns. The galvanometer motor $(6220 \mathrm{H})$ is a closed-loop galvanometer with an opening angle of $\pm 20^{\circ}$ (rated angular excursion: $40^{\circ}$ ), a rotor inertia of $0.125 \mathrm{gm} \cdot \mathrm{cm}^{2}, \pm 10 \%$, a torque constant of $6.17 \times$ $10^{4}$ dyne $\mathrm{cm} / \mathrm{amp}, \pm 10 \%$, a small angle step response time of $200 \mu$ s with a $5 \mathrm{~mm}$ Y mirror, and a linearity of $99.5 \%$. The galvanometer motor $(6220 \mathrm{H})$ used in this work is shown in Fig. 3(a). 


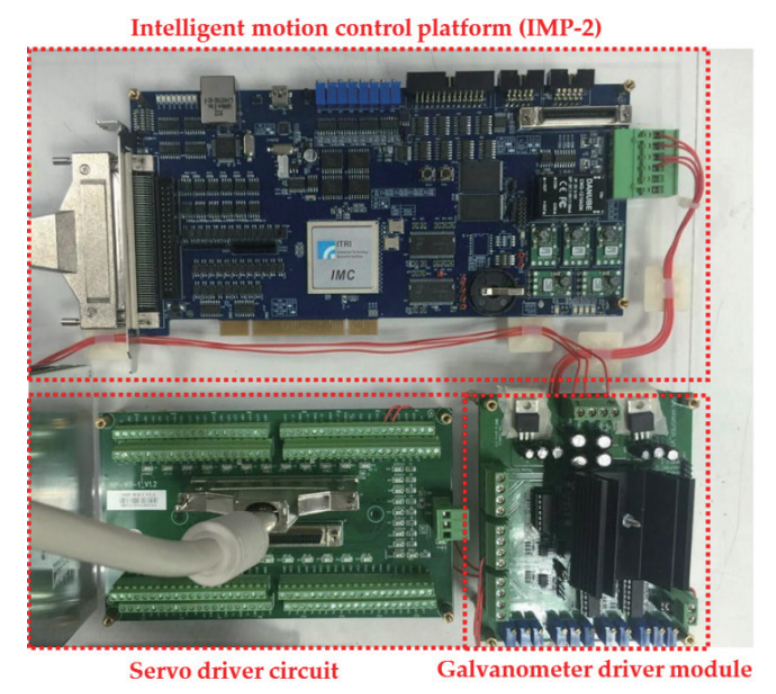

Fig. 2. (Color online) Architecture of the proposed galvanometer control system.

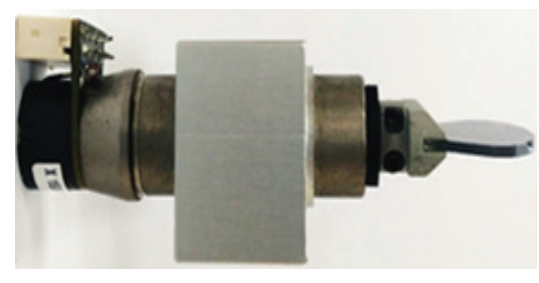

(a)

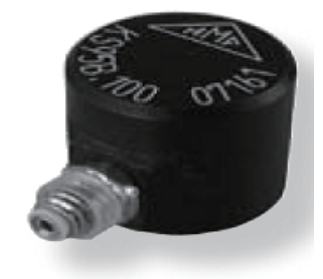

(b)

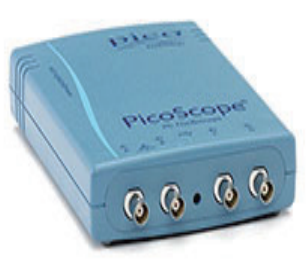

(c)

Fig. 3. (Color online) (a) Galvanometer motor (6220H) with $5 \mathrm{~mm}$ mirror. (b) MEMS piezoelectric accelerometer (KS95B100). (c) Digital oscilloscope (PicoScope 4224 IEPE).

\section{Vibration Measurement, Analysis, and Suppression System}

\subsection{Vibration measurement and analysis system}

The vibration measurement and analysis system proposed in this paper is composed of a MEMS piezoelectric accelerometer (KS95B100) and a notebook computer operating the FFT algorithm. In addition, an oscilloscope (DS4024) is used to measure the position signals of the galvanometer motor. We describe each instrument in detail as follows: (1) MEMS piezoelectric accelerometer (KS95B100): The MEMS-based accelerometer produced by Metra Mess-und Frequenztechnik (Radebeul, Germany) is used for measuring the vibration signal generated from the galvanometer motor $(6220 \mathrm{H})$ during rotation motions. The MEMS-based accelerometer possesses a measurement range of $\pm 60 \mathrm{~g}$, a sensitivity of $100 \pm 5 \% \mathrm{mV} / \mathrm{g}$, a linear frequency range of $0.5-28 \mathrm{kHz}$ at $3 \mathrm{~dB}$, an operation current of 2-20 $\mathrm{mA}$, and an operation temperature of $-20-120{ }^{\circ} \mathrm{C}$. In addition, to record the measurements of the MEMS-based 
accelerometer, we use a 12-bit and 2-channel digital oscilloscope (PicoScope 4224 IEPE, Pico Technology, Cambridgeshire, UK), which has an input range of $\pm 50 \mathrm{mV}- \pm 20 \mathrm{~V}$, a resolution of $12 \mathrm{bits}$, a maximum sampling rate of $80 \mathrm{MS} / \mathrm{s}$, a sensitivity of $10 \mathrm{mV} / \mathrm{div}-4 \mathrm{~V} / \mathrm{div}$, and an operation temperature of $0-45^{\circ} \mathrm{C}$. The digital oscilloscope can transmit the measurements to a notebook computer through a universal series bus (USB) for further vibration analysis. The MEMS-based accelerometer (KS95B100) and digital oscilloscope (PicoScope 4224 IEPE) used in this work are shown in Figs. 3(b) and 3(c), respectively. (2) Notebook computer: The vibration spectrum analysis of the mirror-installed galvanometer motor was performed on a notebook computer running Microsoft Windows 10 operating system with a Core i5-4460 3.2 GHz CPU and 8 GB memory. The vibration spectrum analysis can be executed by MATLAB programs to carry out the FFT analysis of the vibration acceleration signals for determining harmonic or resonance frequencies. (3) Oscilloscope (DS4024): The oscilloscope (DS4024) produced by RIGOL is utilized to measure the position signals of the galvanometer motor and execute the FFT calculation. The oscilloscope is equipped with four analog channels for simultaneous measurement with a bandwidth of $200 \mathrm{MHz}$, a vertical sensitivity of $1 \mathrm{mV} / \mathrm{div}-10 \mathrm{~V} / \mathrm{div}$, and a resolution of 8 bits. The sampling frequency in the real-time waveform analysis mode is $4 \mathrm{GSa} /$ $\mathrm{s}$ with memory depth as many as 1.4 billion points.

The vibration measurement and analysis process of the mirror-installed galvanometer motor is shown in Fig. 4. First, the waveforms and frequencies of the command signals for the galvanometer motor control are set up by the notebook computer and transmitted to the IMP2. Second, the IMP2 generates the digital command signals based on the defined command signals transmitted from the notebook computer. Third, the servo driver circuit receives the digital command signals transmitted from the IMP2 and converts them into the analog command voltage signals. Subsequently, the analog command voltage signals are transmitted to the PID controller and motor driving circuit embedded in the galvanometer driver module for controlling the rotation angles of the scanning mirror installed on the galvanometer

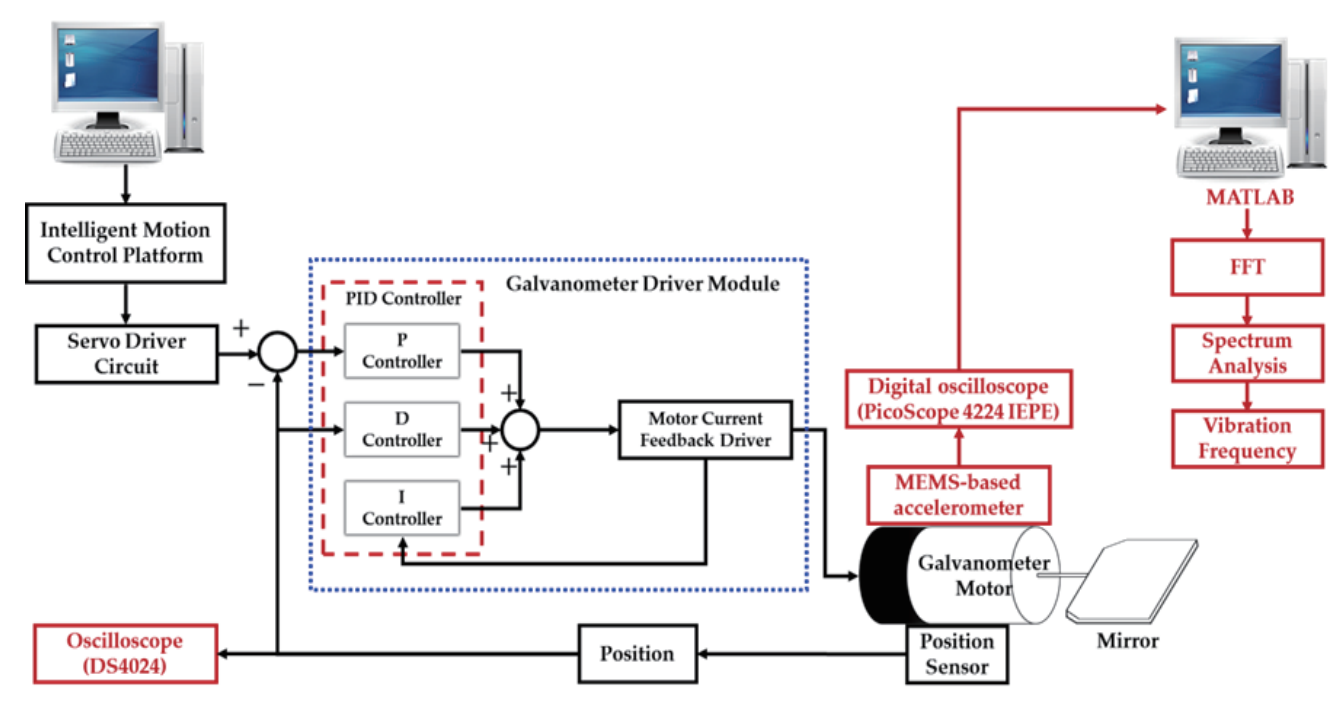

Fig. 4. (Color online) Vibration measurement and analysis process of the mirror-installed galvanometer motor. 
motor. Then, the oscilloscope (DS4024) is used to measure the output position signals of the galvanometer motor and execute the FFT analysis of the output position signals. Finally, the MEMS-based accelerometer and digital oscilloscope are utilized to measure and record the vibration acceleration signals of the mirror-installed galvanometer motor and transmit them to the notebook computer for further FFT spectrum analysis via the MATLAB programs.

\subsection{Vibration suppression circuit design}

In this study, we design an analog comb filter circuit, which connects active Twin-T notch filter circuits to each other in series based on the harmonic frequencies determined by the FFT spectrum analysis of the vibration acceleration signals measured using the MEMS-based accelerometer. Once harmonic frequencies are found, multiple notch filter circuits are designed and connected to each other in series to form a comb filter circuit for suppressing the vibrations of the mirror-installed galvanometer motor. The active Twin-T notch filter circuit utilized in this study is shown in Fig. 5. The state equations for the active Twin-T notch filter are derived as follows. ${ }^{(18)}$

$$
\left\{\begin{array}{c}
\frac{d v_{n 1}(t)}{d t}=Q \frac{d v_{i}(t)}{d t}+\frac{0.5 v_{i}(t)+\left(2 Q^{2}-2 Q+0.5\right) v_{o}(t)+(2 Q-1) v_{n 1}(t)-2 Q v_{n 2}(t)}{R_{n} C_{n}} \\
\frac{d v_{n 2}(t)}{d t}=\frac{d v_{i}(t)}{d t}-\frac{2 v_{n 2}(t)-(2 Q-1) v_{o}(t)-v_{n 1}(t)}{R_{n} C_{n}} \\
\frac{d v_{o}(t)}{d t}=\frac{d v_{i}(t)}{d t}-\frac{2 v_{n 2}(t)-2(Q-1) v_{o}(t)-2 v_{n 1}(t)}{R_{n} C_{n}}
\end{array}\right.
$$

Here, $v_{i}(t)$ and $v_{o}(t)$ are the input and output voltages in the filter circuit, respectively, $v_{n 1}(t)$ and $v_{n 2}(t)$ are the internal state variables in the filter circuit, and $Q$ is the quality factor that can be calculated using $R_{Q 1}$ and $R_{Q 2}$ as follows.

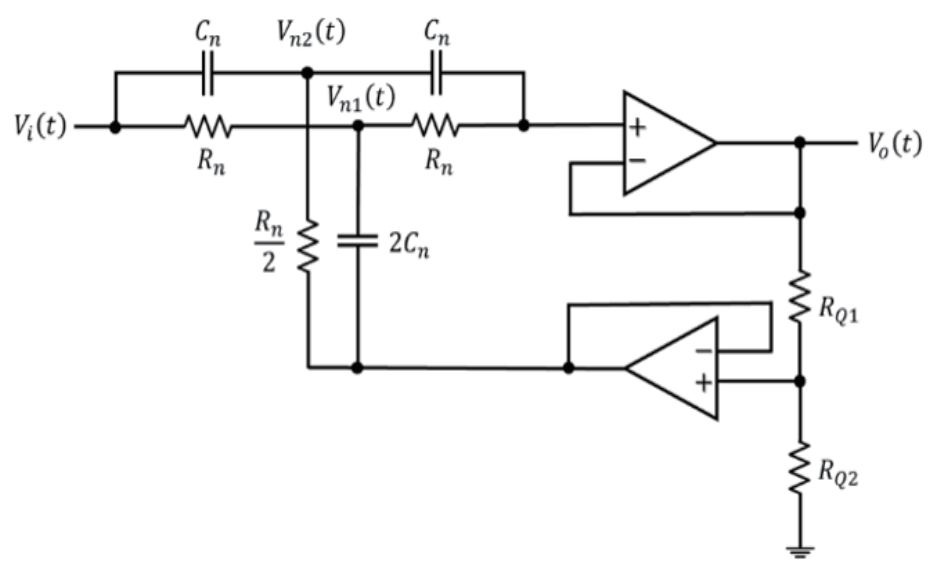

Fig. 5. Active Twin-T notch filter. 


$$
Q=\frac{R_{Q 1}+R_{Q 2}}{4 R_{Q 1}}
$$

By using the Laplace transform, we can obtain the input-output transform function $[T(s)]$ :

$$
T(s)=\frac{V_{o}(s)}{V_{i}(s)}=\frac{s^{2}+\frac{1}{R_{n}^{2} C_{n}^{2}}}{s^{2}+\frac{4}{R_{n} C_{n}}(1-Q) s+\frac{1}{R_{n}^{2} C_{n}^{2}}} .
$$

By letting Eq. (3) equal to zero, the notch frequency $\left(f_{n}\right)$ can be calculated as

$$
f_{n}=\frac{1}{2 \pi R_{n} C_{n}} .
$$

Additionally, based on Eq. (3), the bandwidth $(B W)$ of the notch filter is determined by the quality factor $(Q)$ :

$$
B W=\frac{\frac{1}{R_{n} C_{n}}}{Q} .
$$

When the quality factor $(Q)$ is equal to 1 , the transform function $[T(s)]$ is equal to 1 and the bandwidth $(B W)$ of the notch filter becomes narrow. In contrast, the bandwidth $(B W)$ of the notch filter becomes wider when the quality factor $(Q)$ decreases. When we set $R_{n}=4.87$ $\mathrm{k} \Omega, C_{n}=10 \mathrm{nF}, R_{Q 1}=3.5 \mathrm{k} \Omega$, and $R_{Q 2}=80 \mathrm{k} \Omega$ in the notch filter circuit, we can obtain the quality factor $Q=5.964$, the notch frequency $f_{n}=3.272 \mathrm{kHz}$, and the bandwidth $B W=615$ Hz. The Bode plot of the transform function of the notch filter is shown in Fig. 6. The analog comb filter circuit is shown in Fig. 7. An overview of the vibration measurement, analysis, and suppression system for measuring, analyzing, and suppressing the vibrations of the mirror-
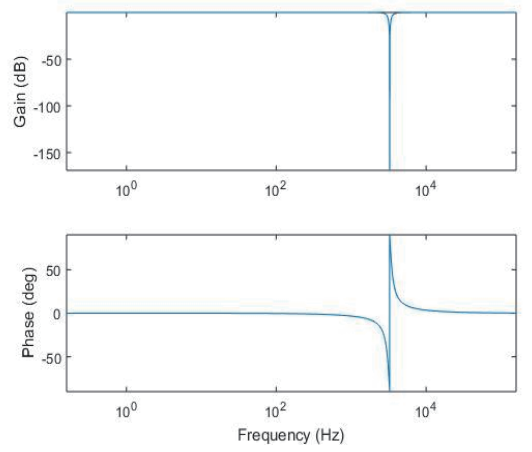

Fig. 6. (Color online) Bode plots of the active Twin-T notch filter.

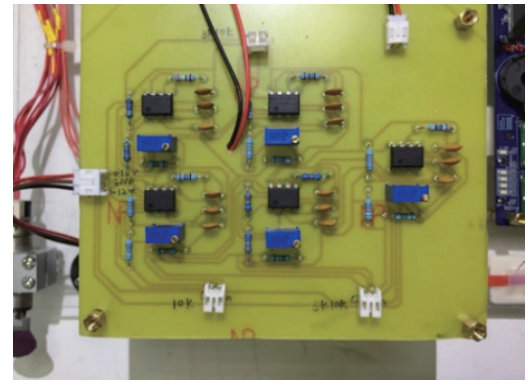

Fig. 7. (Color online) Actual analog comb filter circuit. 
installed galvanometer motor is illustrated in Fig. 8. Once the comb filter circuit is designed on the basis of the harmonic frequencies of the vibration acceleration signals, we can connect it between the PID controller and the motor driving circuit embedded in the galvanometer driver module to suppress the vibrations resulting from the mirror-installed galvanometer motor. The block diagram of the galvanometer control system with the vibration suppression circuit is shown in Fig. 9.

\section{Experimental Results}

In this study, we evaluate the influence of the vibrations on the mirror-installed galvanometer motor with/without analog comb filter circuits using analog command voltage inputs with respect to sine waveforms with different frequencies. First, we drive the mirror-

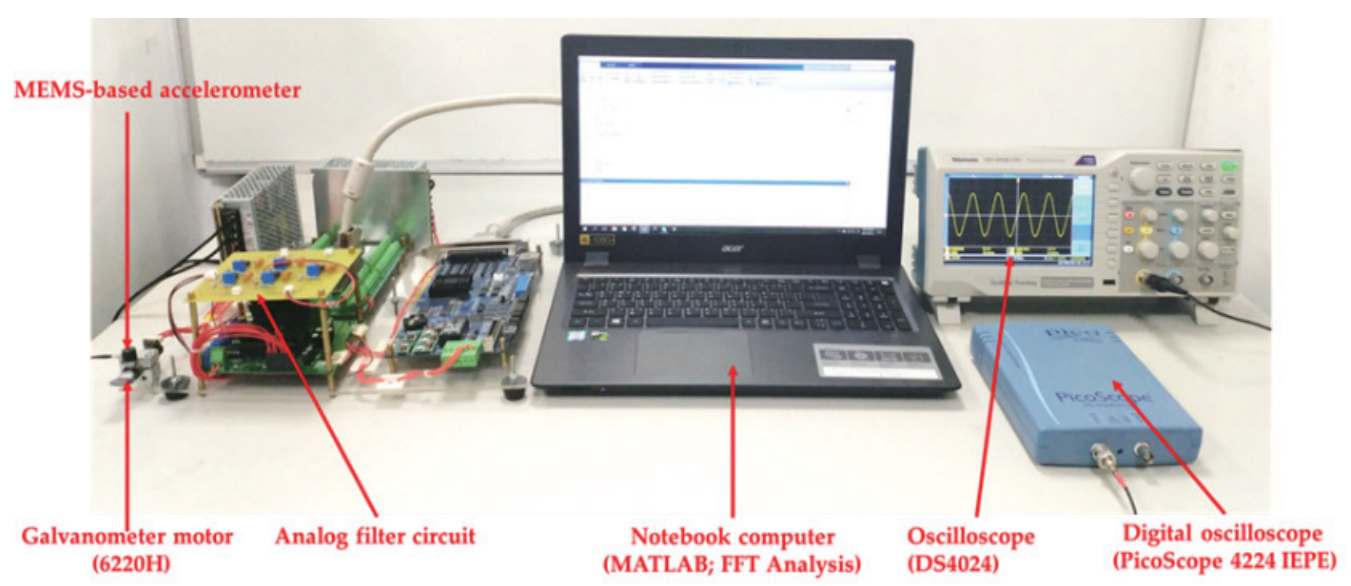

Fig. 8. (Color online) Architecture of the vibration measurement, analysis, and suppression system.

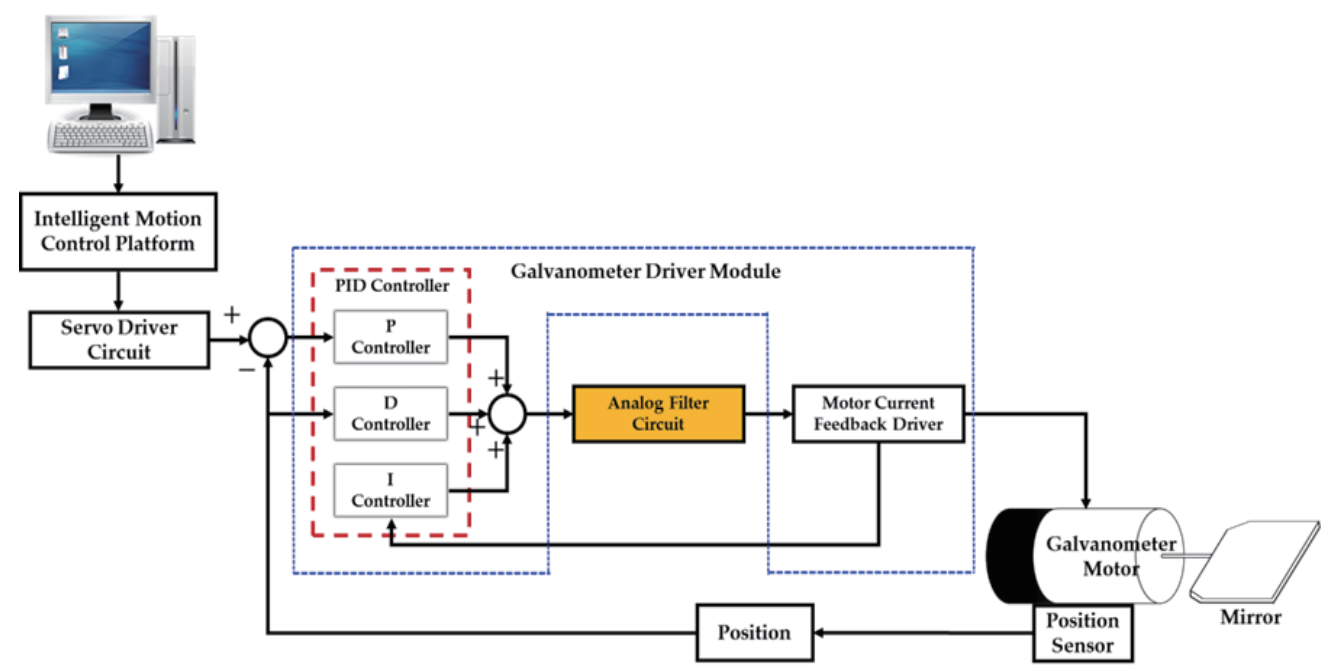

Fig. 9. (Color online) Block diagram of the galvanometer control system with the vibration suppression circuit. 
installed galvanometer motor using two analog command voltage inputs such as 50 and 200 $\mathrm{Hz}$ sine waves for observing the vibration phenomena of the galvanometer motor. Afterwards, the vibration acceleration signals generated from the galvanometer motor are measured using the MEMS-based accelerometer. Subsequently, the FFT algorithm is applied to analyze the vibration spectrum and estimate the harmonic frequencies through the vibration acceleration signals. Then, an analog comb filter is designed with the knowledge of vibration harmonic locations. Finally, the performance of vibration suppression using the designed analog comb filter circuit is evaluated under different operating conditions. In addition, we also use a microphone to measure the sounds of the acoustic noise resulting from the vibration motions of the galvanometer motor, and utilize the FFT to analyze their frequency spectrum to validate the effectiveness of the proposed vibration suppression strategy.

\subsection{Case 1: $55 \mathrm{~Hz}$ sine wave voltage input}

\subsubsection{Vibration signal analysis}

Figure 10(a) shows the time-domain position signal and spectrum of the mirror-installed galvanometer motor under a $55 \mathrm{~Hz}$ sine wave voltage input. A $55.55 \mathrm{~Hz}$ position signal is observed in the DS4024 oscilloscope. In addition, Fig. 10(b) shows the vibration acceleration signals through the measurement using the MEMS-based accelerometer. According to the FFT calculation of the measured vibration acceleration signals, the magnitude responses are shown in Fig. 10(c). Obviously, five frequencies are clearly observed at $55.55 \mathrm{~Hz}, 3.111 \mathrm{kHz}, 5.056$ $\mathrm{kHz}, 10.060 \mathrm{kHz}$, and $15.060 \mathrm{kHz}$. Hence, we can clearly observe that the measured vibration

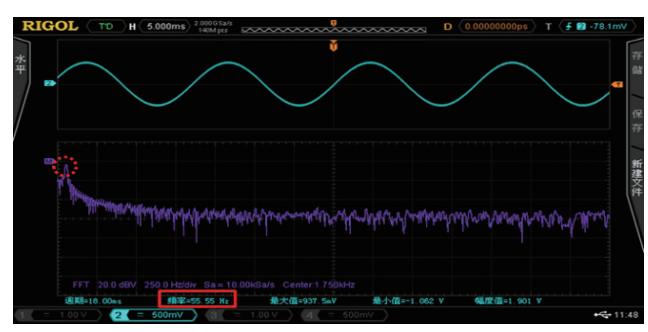

(a)

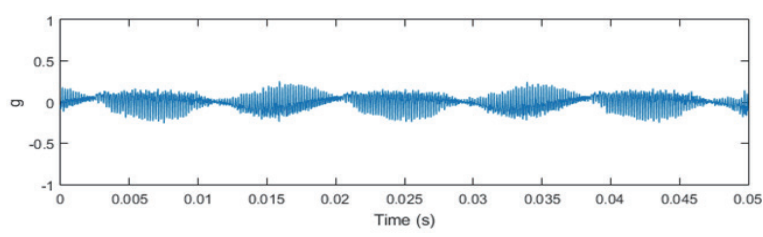

(b)
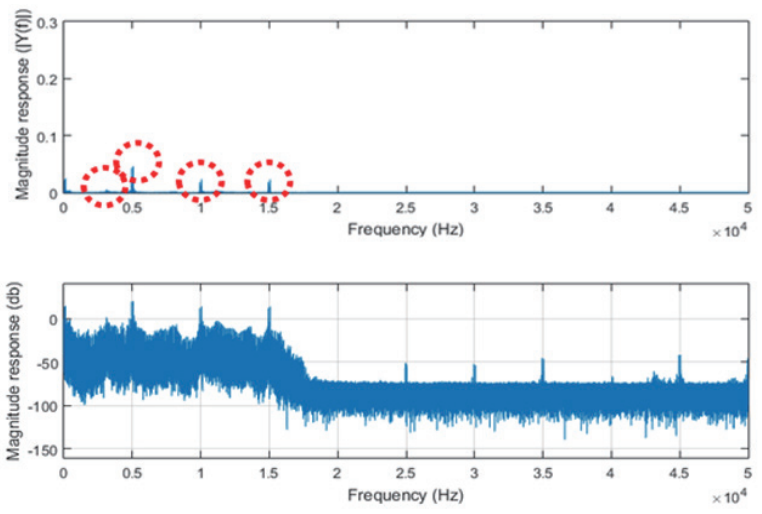

(c)

Fig. 10. (Color online) $55 \mathrm{~Hz}$ sine wave voltage input. (a) Time-domain position signal and spectrum of the mirrorinstalled galvanometer motor measured and analyzed using the DS4024 oscilloscope. (b) Vibration acceleration signals measured using the MEMS-based accelerometer. (c) Magnitude response of the vibration acceleration signals. 
acceleration signals contain not only the $55.55 \mathrm{~Hz}$ frequency resulting from the $55 \mathrm{~Hz}$ sine wave voltage input, but also the other frequencies of 3.111, 5.056, 10.060, and $15.060 \mathrm{kHz}$ generated from the vibration motions of the mirror-installed galvanometer motor.

\subsubsection{Analog filter design}

As shown in Fig. 10, the galvanometer motor contains the vibration harmonics at 3.111, 5.056, 10.060, and $15.060 \mathrm{kHz}$. However, according to our experimental result, the harmonic at 15.060 $\mathrm{kHz}$ is clearly the secondary harmonic resulting from the fundamental harmonic of $5.056 \mathrm{kHz}$. Thus, these two harmonics can be suppressed by filtering the harmonic at $5.056 \mathrm{kHz}$. Hence, analog notch filters are designed with three cut-off frequencies of $3.111,5.056$, and $10.060 \mathrm{kHz}$ to reduce the influence of vibrations on the galvanometer motor. On the basis of the software calculation using OrCAD Capture Lite, the notch and cut-off frequencies of the designed notch filters are listed in Table 1. For the suppression of the first vibration harmonic at $3.111 \mathrm{kHz}$, the first notch filter circuit with $R_{n}=4.87 \mathrm{k} \Omega, C_{n}=10 \mathrm{nF}, R_{Q 1}=3.5 \mathrm{k} \Omega$, and $R_{Q 2}=80 \mathrm{k} \Omega$ is designed to achieve a $3.272 \mathrm{kHz}$ notch frequency with cut-off frequencies of 2.963 and 3.578 $\mathrm{kHz}$. In addition, for the second vibration harmonic at $5.056 \mathrm{kHz}, R_{n}=3.24 \mathrm{k} \Omega, C_{n}=10 \mathrm{nF}$, $R_{Q 1}=3.5 \mathrm{k} \Omega$, and $R_{Q 2}=80 \mathrm{k} \Omega$ are selected in the second notch filter circuit to have a 4.933 $\mathrm{kHz}$ notch frequency with cut-off frequencies of 4.480 and $5.371 \mathrm{kHz}$. For the third vibration harmonic at $10.060 \mathrm{kHz}, R_{n}=1.62 \mathrm{k} \Omega, C_{n}=10 \mathrm{nF}, R_{Q 1}=3.5 \mathrm{k} \Omega$, and $R_{Q 2}=80 \mathrm{k} \Omega$ are used in the third notch filter circuit, resulting in a $9.775 \mathrm{kHz}$ notch frequency with cut-off frequencies of 9.034 and $10.672 \mathrm{kHz}$. Finally, these three analog notch filter circuits are connected to each other in series to formulate an analog comb filter circuit for filtering the vibration harmonics at 3.111, $5.056,10.060$, and $15.060 \mathrm{kHz}$. The schematic of the designed comb filter circuit considering a $55 \mathrm{~Hz}$ sine wave voltage input is shown in Fig. 11 and its actual circuit board is shown in Fig. 7. The Bode plot of the designed comb filter is shown in Fig. 12.

Figure 13 demonstrates the experimental results of the measured time-domain vibration acceleration signals and spectrum after adding the analog comb filter circuit. Obviously, the acceleration harmonics at 3.111, 5.056, 10.060, and $15.060 \mathrm{kHz}$ shown in Fig. 10 are all removed after filtering. From Fig. 10, the time-domain magnitude of the vibration acceleration signal is from -0.256 to $0.252 \mathrm{~g}$. After filtering, the time-domain magnitude of the vibration acceleration signal is from -0.110 to $0.177 \mathrm{~g}$, as shown in Fig. 13. Obviously, the vibration magnitude range

Table 1

Circuit parameters for the designed comb filter under a $55 \mathrm{~Hz}$ sine wave voltage input.

\begin{tabular}{lccc}
\hline Vibration frequency $(\mathrm{kHz})$ & 3.111 & 5.056 & 10.060 \\
\hline Notch frequency $(\mathrm{kHz})$ & 3.272 & 4.933 & 9.775 \\
Cut-off frequency $(\mathrm{kHz})$ & $2.963,3.578$ & $4.480,5.371$ & $9.034,10.672$ \\
Bandwidth $(\mathrm{Hz})$ & 615 & 891 & 1638 \\
$R_{n}(\mathrm{k} \Omega)$ & 4.870 & 3.240 & 1.620 \\
$R_{Q 1}(\mathrm{k} \Omega)$ & 3.500 & 3.500 & 3.500 \\
$R_{Q 2}(\mathrm{k} \Omega)$ & 80 & 80 & 80 \\
$C_{n}(\mathrm{nF})$ & 10 & 10 & 10 \\
\hline
\end{tabular}




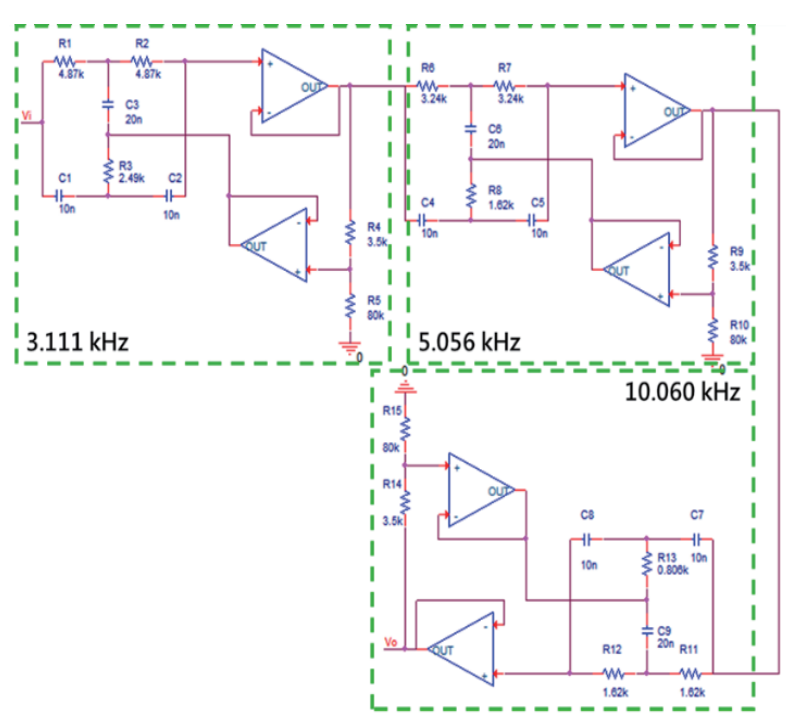

Fig. 11. (Color online) Analog comb filter circuit for suppressing the vibrations generated from the $55 \mathrm{~Hz}$ sine wave voltage input.

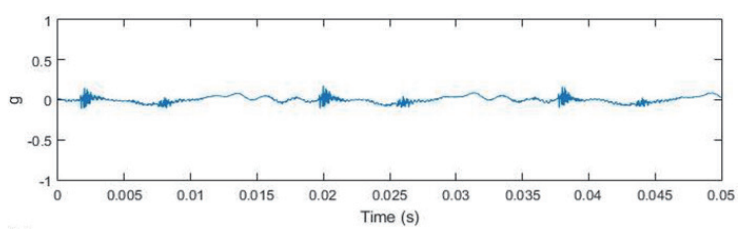

(a)
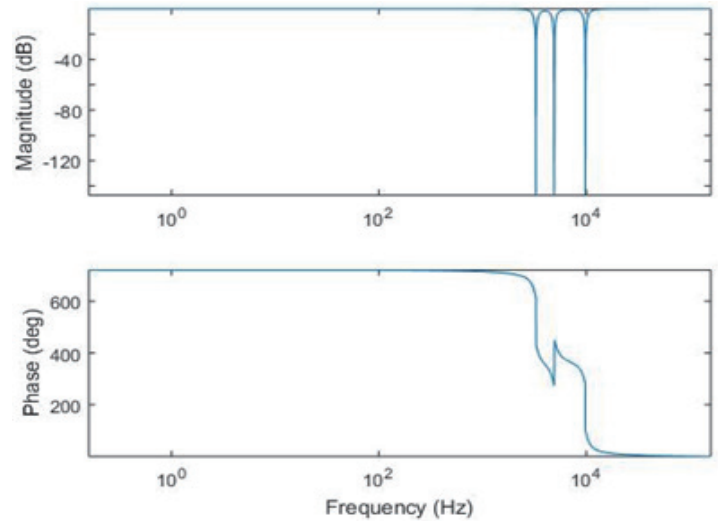

Fig. 12. (Color online) Bode diagram of the comb filter considering a $55 \mathrm{~Hz}$ sine wave voltage input.
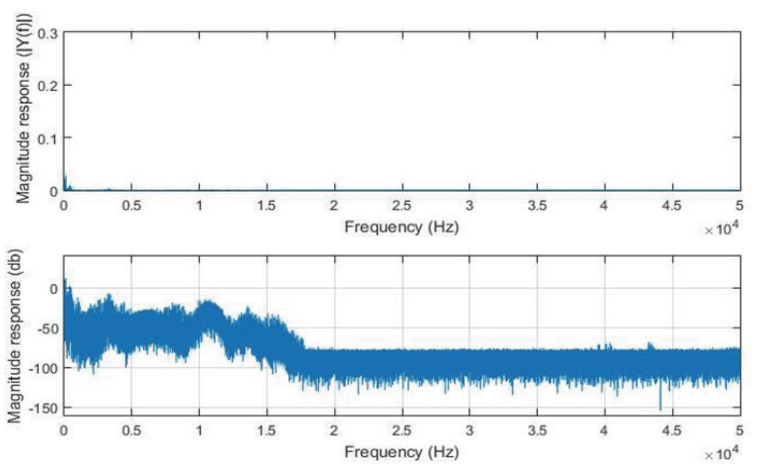

(b)

Fig. 13. (Color online) $55 \mathrm{~Hz}$ sine wave voltage input: (a) Vibration acceleration signals measured using the MEMS-based accelerometer after filtering. (b) Magnitude response of the vibration acceleration signals after filtering.

of the acceleration signal decreases from 0.508 to $0.287 \mathrm{~g}$ after filtering, and the improvement rate of the vibration magnitude is $43.5 \%$.

\subsubsection{Verification by using sound signals}

To validate that the designed analog comb filter circuit can suppress the vibrations of the galvanometer motor effectively, we utilize a microphone to record the noise sounds of the acoustic noise resulting from the vibration motions of the galvanometer motor for the frequency spectrum analysis. Figures 14 and 15 show both the time-domain acoustic noise amplitude and spectrum before and after adding the comb filter under a $55 \mathrm{~Hz}$ sine wave voltage input. As 


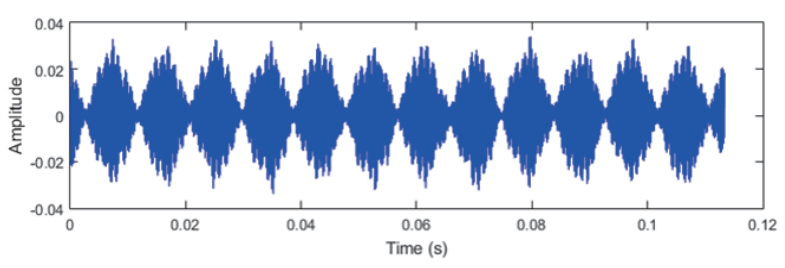

(a)
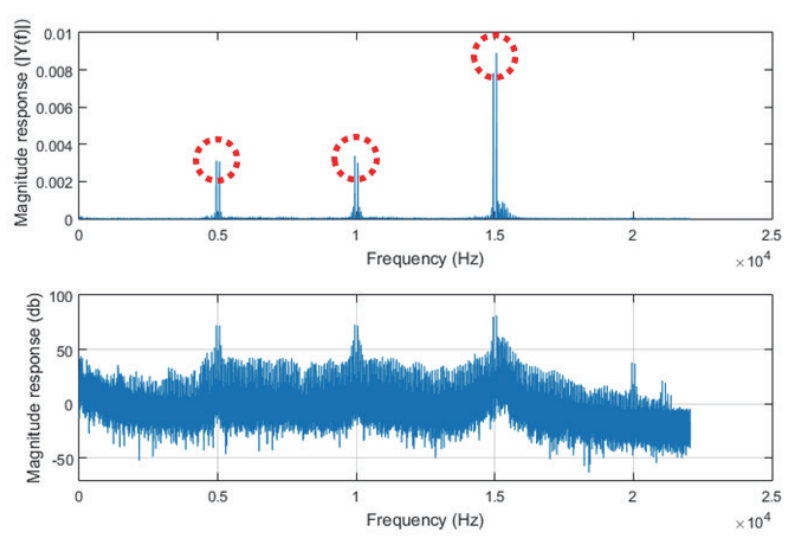

(b)

Fig. 14. (Color online) $55 \mathrm{~Hz}$ sine wave voltage input. (a) Time-domain acoustic noise amplitude measured using the microphone before filtering. (b) Magnitude response of the acoustic noise before filtering.

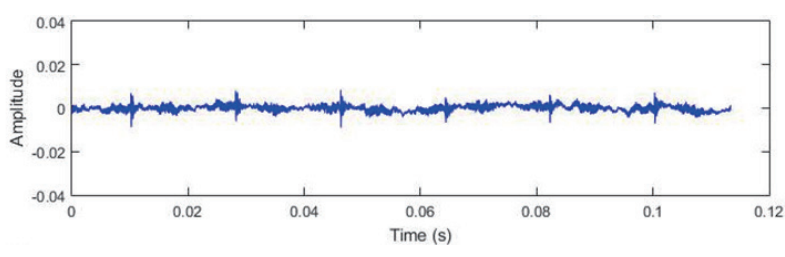

(a)
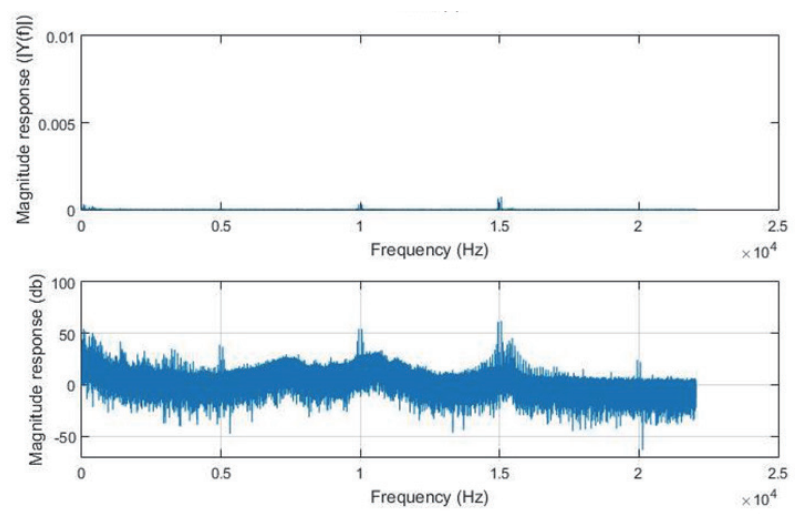

(b)

Fig. 15. (Color online) $55 \mathrm{~Hz}$ sine wave voltage input. (a) Time-domain acoustic noise amplitude measured using the microphone after filtering. (b) Magnitude response of the acoustic noise after filtering.

shown in the noise spectrum in Fig. 14 without the comb filter, noise harmonics at $4.944 \mathrm{~Hz}, 5.055$ $\mathrm{kHz}, 9.944 \mathrm{kHz}, 10.060 \mathrm{kHz}, 14.940 \mathrm{kHz}$, and $15.060 \mathrm{kHz}$ are clearly observed. By contrast, Fig. 15 shows the noise spectrum where the designed comb filter is added. All the magnitudes of harmonics at the same frequencies decrease with the help of the analog comb filter.

\subsection{Case 2: $220 \mathrm{~Hz}$ sine wave voltage input}

\subsubsection{Vibration signal analysis}

Figure 16(a) shows the time-domain position signal and spectrum of the mirror-installed galvanometer motor under a $220 \mathrm{~Hz}$ sine wave voltage input. A $223.2 \mathrm{~Hz}$ position signal is observed in the DS4024 oscilloscope. In addition, Fig. 16(b) shows the vibration acceleration signals through the measurement using the MEMS-based accelerometer. According to the FFT 


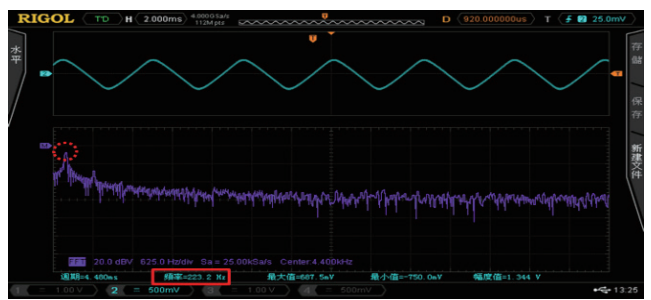

(a)

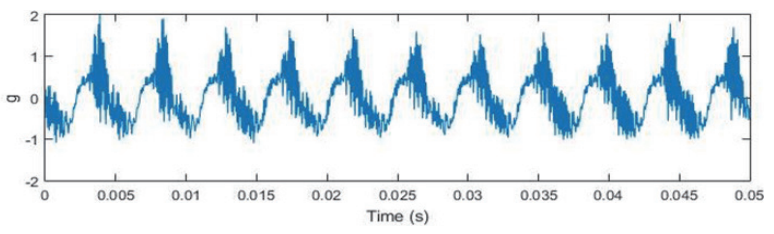

(b)
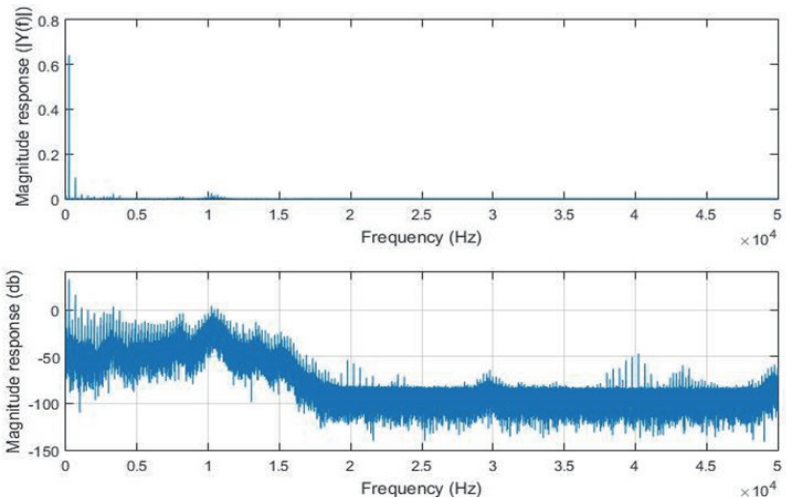

(c)

Fig. 16. (Color online) $220 \mathrm{~Hz}$ sine wave voltage input. (a) Time-domain position signal and spectrum of the mirror-installed galvanometer motor measured and analyzed using the DS4024 oscilloscope. (b) Vibration acceleration signals measured using the MEMS-based accelerometer. (c) Magnitude response of the vibration acceleration signals.

calculation of the measured vibration acceleration signals, the magnitude responses are shown in Fig. 16(c). Obviously, three frequencies are clearly observed at $221.5 \mathrm{~Hz}, 3.331 \mathrm{kHz}$, and $10.220 \mathrm{kHz}$. Hence, we can clearly observe that the measured vibration acceleration signals contain not only the $221.5 \mathrm{~Hz}$ frequency resulting from the $220 \mathrm{~Hz}$ sine wave voltage input, but also other frequencies of 3.331 and $10.220 \mathrm{kHz}$ generated from the vibration motions of the mirror-installed galvanometer motor.

\subsubsection{Analog filter design}

As shown in Fig. 16, the galvanometer motor contains the vibration harmonics at 3.331 and $10.220 \mathrm{kHz}$. Hence, analog notch filters are designed with two cut-off frequencies of 3.331 and $10.220 \mathrm{kHz}$ to reduce the influence of vibration on the galvanometer motor. On the basis of the software calculation using OrCAD Capture Lite, the notch and cut-off frequencies of the designed notch filters are listed in Table 2. For the suppression of the first vibration harmonic at $3.331 \mathrm{kHz}$, the first notch filter circuit with $R_{n}=4.87 \mathrm{k} \Omega, C_{n}=10 \mathrm{nF}, R_{Q 1}=3.5 \mathrm{k} \Omega$, and $R_{Q 2}=80 \mathrm{k} \Omega$ is designed to achieve a $3.272 \mathrm{kHz}$ notch frequency with cut-off frequencies of 2.963 and $3.578 \mathrm{kHz}$. For the second harmonic at $10.220 \mathrm{kHz}, R_{n}=1.62 \mathrm{k} \Omega, C_{n}=10 \mathrm{nF}, R_{Q 1}$ $=3.5 \mathrm{k} \Omega$, and $R_{Q 2}=80 \mathrm{k} \Omega$ are used in the second notch filter circuit, resulting in a $9.775 \mathrm{kHz}$ notch frequency with cut-off frequencies of 9.034 and $10.672 \mathrm{kHz}$. Finally, these two RC notch filter circuits are connected to each other in series to form an analog comb filter for filtering the vibration harmonics at 3.331 and $10.220 \mathrm{kHz}$. The schematic of the designed comb filter circuit considering a $220 \mathrm{~Hz}$ sine wave voltage input is shown in Fig. 17 and its actual circuit board is shown in Fig. 7. The Bode plot of the designed comb filter is shown in Fig. 18. 
Table 2

Circuit parameters for the designed comb filter under a $220 \mathrm{~Hz}$ sine wave voltage input.

\begin{tabular}{lcc}
\hline Vibration frequency $(\mathrm{kHz})$ & 3.331 & 10.220 \\
\hline Notch frequency $(\mathrm{kHz})$ & 3.272 & 9.775 \\
Cut-off frequency $(\mathrm{kHz})$ & $2.963,3.578$ & $9.034,10.672$ \\
Bandwidth $(\mathrm{Hz})$ & 615 & 1638 \\
$R_{n}(\mathrm{k} \Omega)$ & 4.870 & 1.620 \\
$R_{Q 1}(\mathrm{k} \Omega)$ & 3.500 & 3.500 \\
$R_{Q 2}(\mathrm{k} \Omega)$ & 80 & 80 \\
$C_{n}(\mathrm{nF})$ & 10 & 10 \\
\hline
\end{tabular}

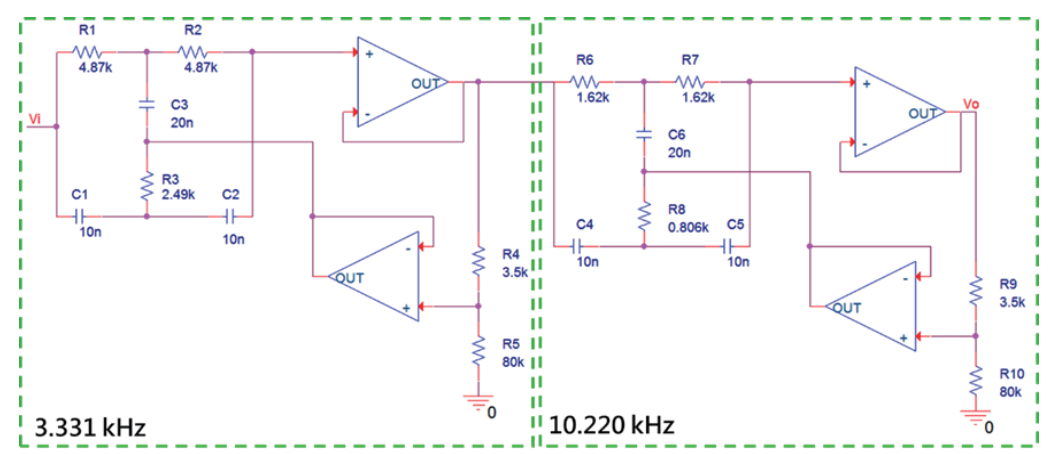

Fig. 17. (Color online) Analog comb filter circuit for suppressing the vibrations generated from the $220 \mathrm{~Hz}$ sine wave voltage input.
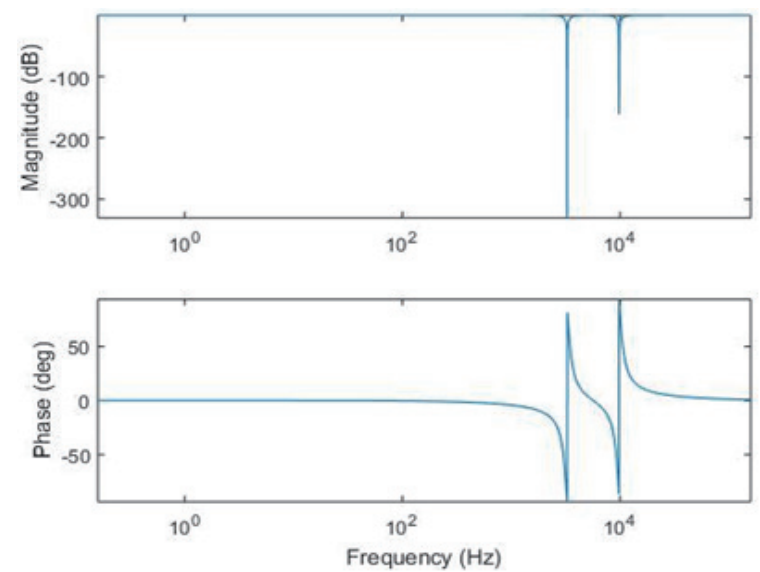

Fig. 18. (Color online) Bode diagram of the comb filter considering a $220 \mathrm{~Hz}$ sine wave voltage input.

Figure 19 demonstrates the experimental results of the measured time-domain vibration acceleration signals and spectrum after adding the analog comb filter circuit. Obviously, the acceleration harmonics at 3.331 and $10.220 \mathrm{kHz}$ shown in Fig. 16 are all removed after filtering. From Fig. 16, the time-domain magnitude of the vibration acceleration signal is from -1.098 to $1.999 \mathrm{~g}$. After filtering, the time-domain magnitude of the vibration acceleration signal is from -0.990 to $0.853 \mathrm{~g}$, as shown in Fig. 19. Obviously, the vibration magnitude range of the acceleration signal decreases from 3.097 to $1.843 \mathrm{~g}$ after filtering, and the improvement rate of the vibration magnitude could achieve $40.5 \%$. 


\subsubsection{Verification by using sound signals}

To validate that the designed analog comb filter circuit can suppress the vibrations of the galvanometer motor effectively, we utilize a microphone to record the noise sounds of the acoustic noise resulting from the vibration motions of the galvanometer motor for the frequency spectrum analysis. Figures 20 and 21 show both the time-domain acoustic noise amplitude and spectrum before and after adding the comb filter under a $220 \mathrm{~Hz}$ sine wave voltage input. As shown in the noise spectrum in Fig. 20 without the comb filter circuit, noise harmonics at 9.998 $\mathrm{Hz}$ and $15.110 \mathrm{kHz}$ are clearly observed. By contrast, Fig. 21 shows the noise spectrum where the designed comb filter is added. All the magnitudes of harmonics at the same frequencies decrease with the help of the analog comb filter.

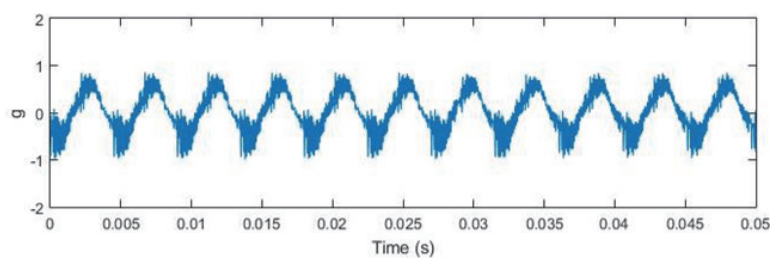

(a)
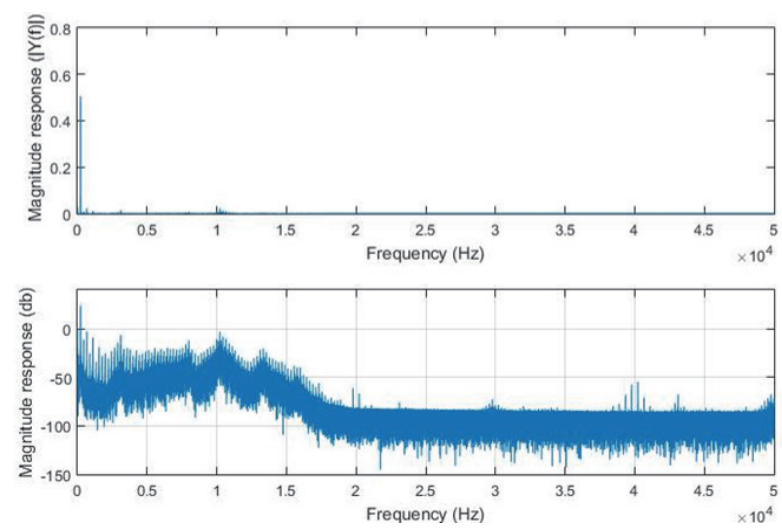

(b)

Fig. 19. (Color online) $220 \mathrm{~Hz}$ sine wave voltage input: (a) Vibration acceleration signals measured using the KS95B100 accelerometer after filtering. (b) Magnitude response of the vibration acceleration signals after filtering.

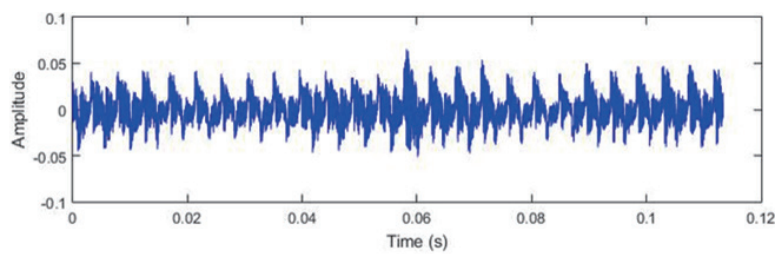

(a)
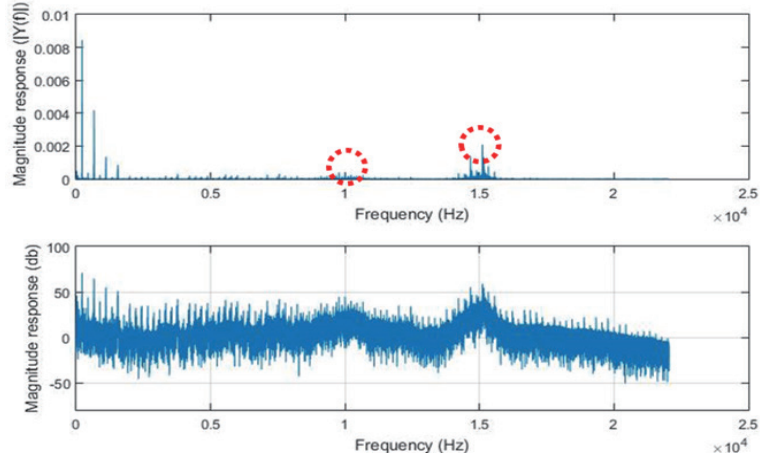

(b)

Fig. 20. (Color online) $220 \mathrm{~Hz}$ sine wave voltage input. (a) Time-domain acoustic noise amplitude measured using the microphone before filtering. (b) Magnitude response of the acoustic noise before filtering. 


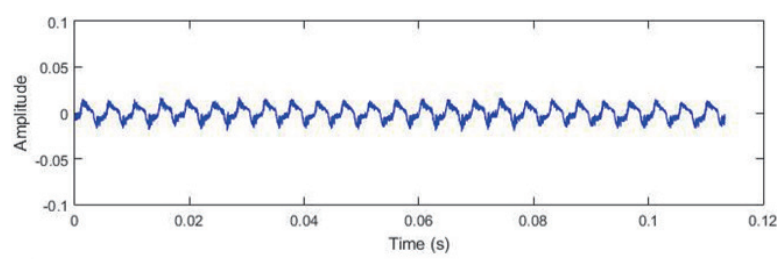

(a)
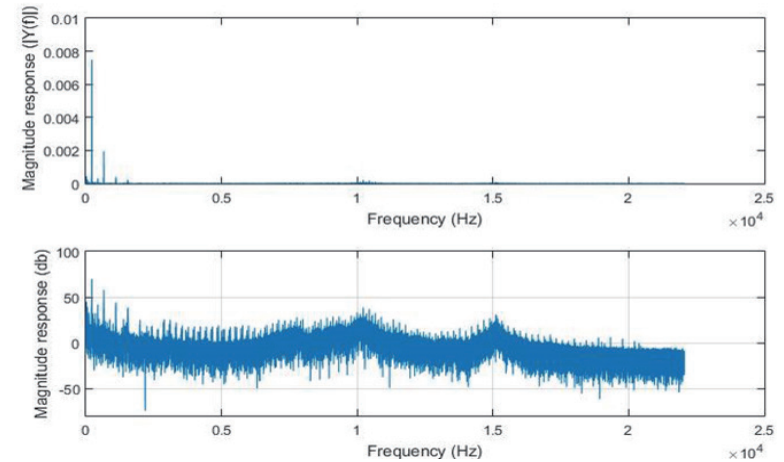

(b)

Fig. 21. (Color online) $220 \mathrm{~Hz}$ sine wave voltage input: (a) Time-domain acoustic noise amplitude measured using the microphone after filtering. (b) Magnitude response of the acoustic noise after filtering.

\section{Conclusions}

In this paper, we present a vibration measurement, analysis, and suppression system for suppressing the vibrations resulting from mirror-installed laser galvanometers. The galvanometer control system integrates a notebook computer, an intelligent motion control platform, a servo driver circuit, and a galvanometer driver module for driving the galvanometers. The proposed vibration measurement, analysis, and suppression system composed of the MEMS-based piezoelectric accelerometer, FFT algorithm, and analog comb filter circuit is utilized to measure, analyze, and suppress vibrations generated from the galvanometers during rotation motions, respectively. The experimental results indicate that the vibration harmonics can be eliminated effectively by utilizing the proposed vibration suppression strategy. After filtering, the vibration magnitude ranges of the acceleration signals decrease from 0.508 to $0.287 \mathrm{~g}$ and from 3.097 to $1.843 \mathrm{~g}$ under the 55 and $220 \mathrm{~Hz}$ sine wave voltage inputs, respectively. The improvement rates of the vibration magnitude are 43.5 and $40.5 \%$ for the 55 and $220 \mathrm{~Hz}$ sine wave voltage inputs, respectively. Additionally, both the time-domain acoustic noise amplitude and spectrum could also validate the effectiveness of the vibration suppression strategy. Finally, the experimental results have successfully validated that the proposed inertial-sensing-based vibration measurement, analysis and suppression system can measure and analyze the galvanometer vibrations, and restrain them effectively to improve the accuracy and stability of the laser galvanometer scanning systems.

\section{Acknowledgments}

This work is supported by the Ministry of Science and Technology of the Republic of China, Taiwan, under Grant Nos. MOST 105-2221-E-035-057, MOST 106-2221-E-035-004, and MOST 106-2813-C-035-036-E, and Industrial Technology Research Institute, Taiwan, R.O.C., under a project with Grant No. H301AR1C30. 


\section{References}

1 Y. Maeda and M. Iwasaki: IEEE Trans. Ind. Electron. 62 (2015) 466. https://doi.org/10.1109/TIE.2014.2331037

2 D. Matsuka, T. Tanaka, and M. Iwasaki: IEEE Trans. Ind. Electron. 63 (2016) 5514. https://doi.org/10.1109/ TIE.2016.2561878

3 S. Chi, Z. Xie, and W. Chen: Sensors 16 (2016) 1534. https://doi.org/10.3390/s16091534

4 J. F. Hsu, S. Dhingra, and B. D’Urso: Am. J. Phys. 85 (2017) 68. https://doi.org/10.1119/1.4972046

5 C. Yu, X. Chen, and J. Xi: Sensors 17 (2017) 164. https://doi.org/10.3390/s17010164

6 C. Lee, A. Zhang, B. Yu, and S. Park: Sensors 17 (2017) 1224. https://doi.org/10.3390/s17061224

7 Z. Xia, S. Xia, L. Wan, and S. Cai: Sensors 12 (2012) 13694. https://doi.org/10.3390/s121013694

8 D. Kim, J. Song, and G. Jang: IEEE Trans. Magn. 50 (2014) 753. https://doi.org/10.1109/TMAG.2013.2285386

9 L. Zhu, B. Wang, R. Yan, Q. Yang, Y. Yang, and X. Zhang: IEEE Trans. Magn. 52 (2016) 1. https://doi. org/10.1109/TMAG.2015.2497738

10 R. Zachar, P. Lindahl, J. Donnal, W. Cotta, C. Schantz, and S. B. Leeb: IEEE Trans. Instrum. Meas. 65 (2016) 1641. https://doi.org/10.1109/TIM.2016.2540944

11 T. K. Chung, P. C. Yeh, H. Lee, C. M. Lin, C. Y. Tseng, W. T. Lo, C. M. Wang, W. C. Wang, C. J. Tu, P. Y. Tasi, and J. W. Chang: Sensors 16 (2016) 269. https://doi.org/10.3390/s16030269

12 G. S.Maruthi and V. Hegde: IEEE Sens. J. 16 (2016) 145. https://doi.org/10.1109/JSEN.2015.2476561

13 C. Y. Huang and J. H. Chen: Appl. Sci. 6 (2016) 201. https://doi.org/10.3390/app6070201

14 R. P. Linessio, K. D. M. Sousa, T. D. Silva, C. A. Bavastri, P. F. D. C. Antunes, and J. C. C. D. Silva: IEEE Sens. J. 16 (2016) 8075. https://doi.org/10.1109/JSEN.2016.2604850

15 J. D. Son, B. H. Ahn, J. M. Ha, and B. K. Choi: Measurement 94 (2016) 680. https://doi.org/10.1016/ j.measurement.2016.08.035

16 C. H. Yang, K. J. Loh, Y. S. Yang, W. Y. Hsiung, and Y. T. Huang: Struct. Control Health Monit. 24 (2017) 1876. https://doi.org/10.1002/stc.1876

17 D. H. Yang, J. H. Shin, H. Lee, S. K. Kim, and M. K. Kwak: J. Sound Vib. 392 (2017) 18. https://doi. org/10.1016/j.jsv.2016.12.036

18 H. H. C. Iu, D. S. Yu, A. L. Fitch, V. Sreeram, and H. Chen: IEEE Trans. Circuits Syst. I Regul. Pap. 58 (2011) 1337. https://doi.org/10.1109/TCSI.2010.2097771 